\title{
CHALLENGES, OPPORTUNITIES, AND SOLUTIONS FOR CONVERGED SATELLITE AND TERRESTRIAL NeTWORKS
}

\author{
TARIK TALEB, NEC EUROPE LTD. \\ YASSINE HADJADJ-AOUL, UNIVERSITY OF RENNES 1 \\ TOUFIK AHMED, UNIVERSITY OF BORDEAUX 1
}

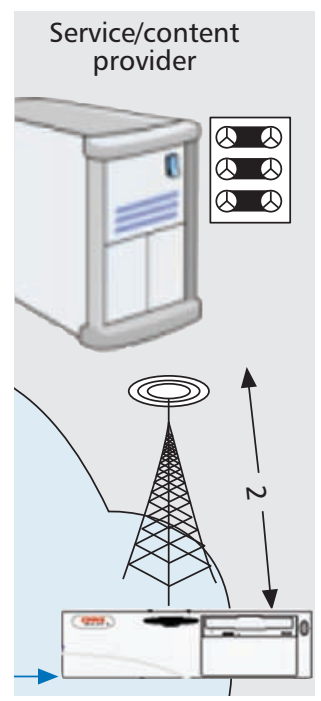

The authors define

some issues related

to the interworking

operation between

the satellite and

terrestrial domains,

and suggest some

solutions and discuss

their potential.

\begin{abstract}
The current trend in telecommunications services provisioning is shifting toward global ubiquitous networking and unified service architecture. Given the diversity of access technologies, this global ubiquitous networking cannot be possible without efficient interworking between the different access players. This leads to the necessity of defining, implementing, and deploying common services control architecture, able to support a wide variety of services for users in a variety of roles (consumer, producer, or manager of communication and media). This article defines some issues related to the interworking operation between the satellite and terrestrial domains. It suggests some solutions and discusses their potential.
\end{abstract}

\section{INTRODUCTION}

Wireless communications continues to pervade all aspects of our lives - wireless distribution of audio and video around the home, wireless solutions for logistics, wireless ticketing and access control, wireless sensors for agriculture, medical applications, and so on. While many people appreciate the profound impact that wireless communications are having and will have on our lives, it will be some time before the vision of wireless everywhere will be realized - mainly because introducing large-scale changes to the way many systems work is complex, and requires significant time, effort, and energy. While there have been many important advances in wireless technology in recent years, there are economic challenges in providing high-speed wireless access to less populated areas. This gap between those who benefit from digital technology and those who do not is known as digital divide.

A key technology that can help to bridge the digital divide is satellite communications as it can be used in areas where there is no terrestrial alternative. In the developed world, satellite net- works can be interworked with existing terrestrial networks, be they wireless or fixed systems, core or access network, and function as a high-speed backbone network to support a wide variety of services for users in a variety of roles.

In this regard, there are many lessons to learn from recent mobile satellite experience. Indeed, in urban/suburban areas, fixed and mobile technologies (e.g., asymmetrick digital subscriber line [ADSL], Global System for Mobile Communications [GSM]) are well advanced. Satellites, performing in isolation, cannot compete with terrestrial systems in these urban areas. They can only provide niche services to areas inaccessible to terrestrial technologies. While these markets are politically important, they are small and bring poor revenue for satellite operators. The future of nextgeneration satellite systems is clearly in an integrated architecture with terrestrial systems. Their success also hinges on their ability to provide, in full cooperation with terrestrial systems, broadband data rate applications similar in spirit to today's Internet. This is also beneficial for terrestrial system operators as it will enable them to increase the capacity of their systems, support large-scale deployment of different emerging bandwidth-intensive services, and satisfy the ever growing community of Internet users.

Two critical issues arise when considering satellite systems in this context. First, satellite systems are very costly in general; second, there are challenges in integrating satellite and terrestrial networks, particularly when terminal mobility is necessary. This article will give some insight toward solving both of these problems.

In this article we focus on interworking between the satellite part of the network and its terrestrial counterpart. Interworking related operations are performed at newly defined entities called interworking gateways (IGWs). The scope of this article is to define the modules of the technological solutions that will be incorpo- 


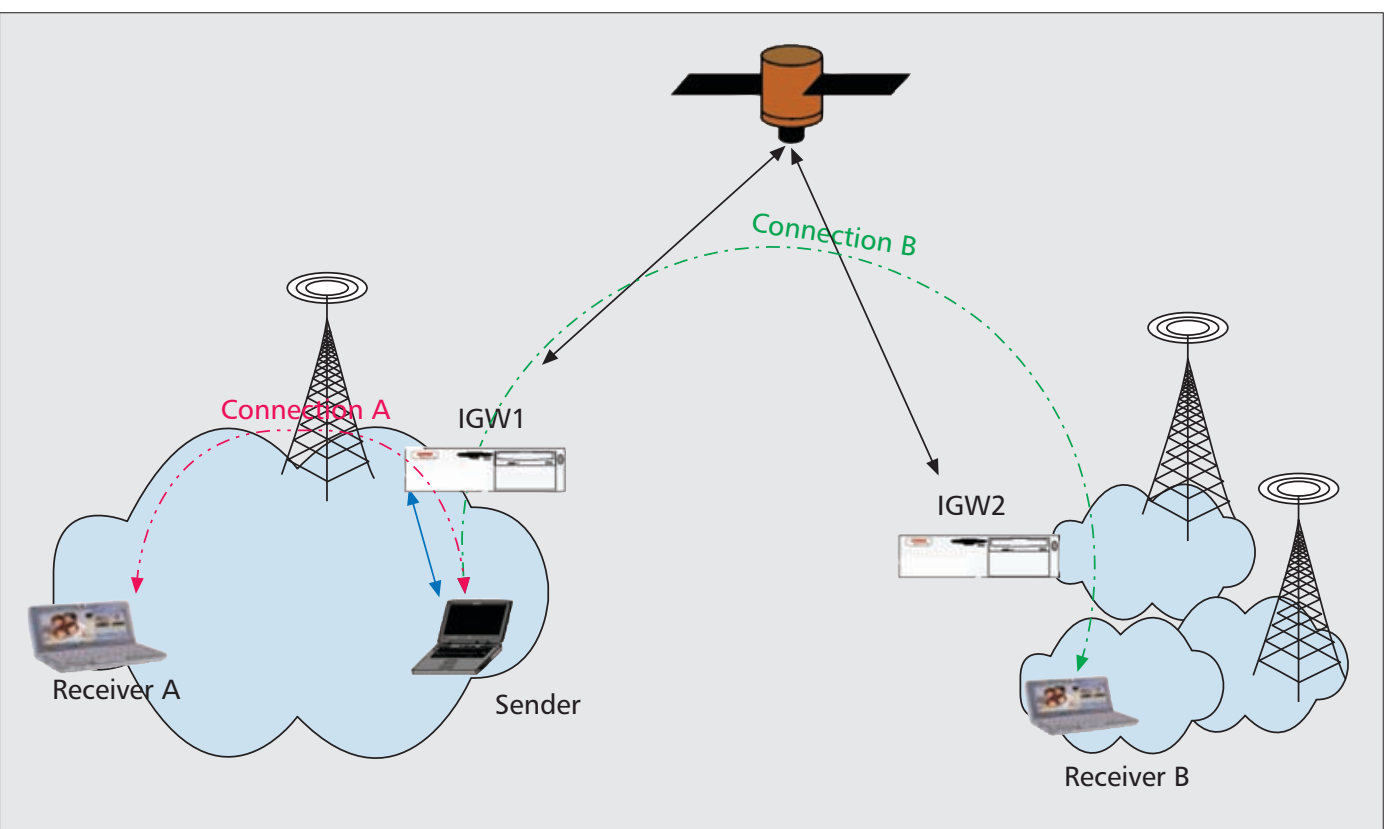

Figure 1. RTT-based connection setup + connection decoupling.

rated in IGWs and evaluate their performances via computer simulations.

The remainder of this article is organized as follows. The next section portrays the key components of the envisioned architecture. We then describe our proposed context-aware complete end-to-end $Q o S$ approach devised for interworking between the satellite and terrestrial domains. The article concludes in the final section.

\section{Envisioned Network Topology}

Although geostationary Earth orbit (GEO) systems are widely in use, and low/medium Earth orbit (LEO/MEO) will come onto the scene in the longer term, this article does not target any particular satellite constellation type. The developed solutions will be designed to be applicable to all constellation types (GEO, MEO, and LEO). The satellites are only assumed to be bidirectional interactive, acquiring onboard processing (OBP) capabilities and intersatellite links (ISLs) [1]. Terminals are interactive. Terminals outside the reach of the terrestrial network have direct access to the satellites. Terminals within reach of the ground Internet infrastructure have the ability to connect either directly to satellites or via the IGWs. The overall objective of this article is to define the necessary intelligence that should be added to IGWs to guarantee context-aware complete end-toend quality of service (QoS) for users. Thus, different levels of convergence are considered. The first level of convergence concerns efficient data transmission based on IP. The second level refers to the control and signaling for providing resource allocation and management. The third level of convergence deals with the provisioning of a generic service delivery platform based in IP Multimedia Subsystem (IMS). Finally, mobility management and seamless connectivity are considered for both network-link handover and link-layer handover.

\section{CONTEXT-AWARE COMPLETE END-TO-END QOS APPROACH \\ EFFicient Data TRANSmission}

First, in light of the rapid globalization of the Internet and the resultant universality of IP, the data traffic load to be generated from the interworked satellite/terrestrial networks is expected to be all-IP as well. Investigating the interactions of IP protocols with the network is of vital importance.

Satellite systems are well known for their unique characteristics - long propagation delays, large delay-bandwidth product, errors due to propagation corruption and handovers, and variable round-trip time (RTT) and link handovers. These features put limitation on the working of most transmission. With this regard, the authors have recently developed the Recursive, Explicit, and Fair Window Adjustment (REFWA) method to enhance the efficiency and fairness of TCP in satellite systems [2]. The use of the REFWA scheme has been extended further to the case of hybrid wired/wireless networks as well. While the REFWA scheme exhibits good performance, its performance remains limited in large bandwidth environments (such as satellite systems) due to its windowbased nature. Development of a new rate-based congestion control protocol that is specifically tailored for satellites and can exploit well the large delay bandwidth product feature of the satellite systems is required. REFWA can be a good candidate for that by changing its window-based feature to a rate-based one. Indeed, this is possible by having IGWs send data at rates exactly equal to the feedback value computed by REFWA. This is similar in spirit to the concept of the Explicit Control Protocol (XCP). Using such rate-based congestion control mechanism, and similar in spirit to the connection splitting in [3], the full end-to-end path will be decoupled into from the interworked satellite/terrestrial networks is expected to be all-IP as well. Investigating the interactions of IP protocols with the network is of vital importance. 


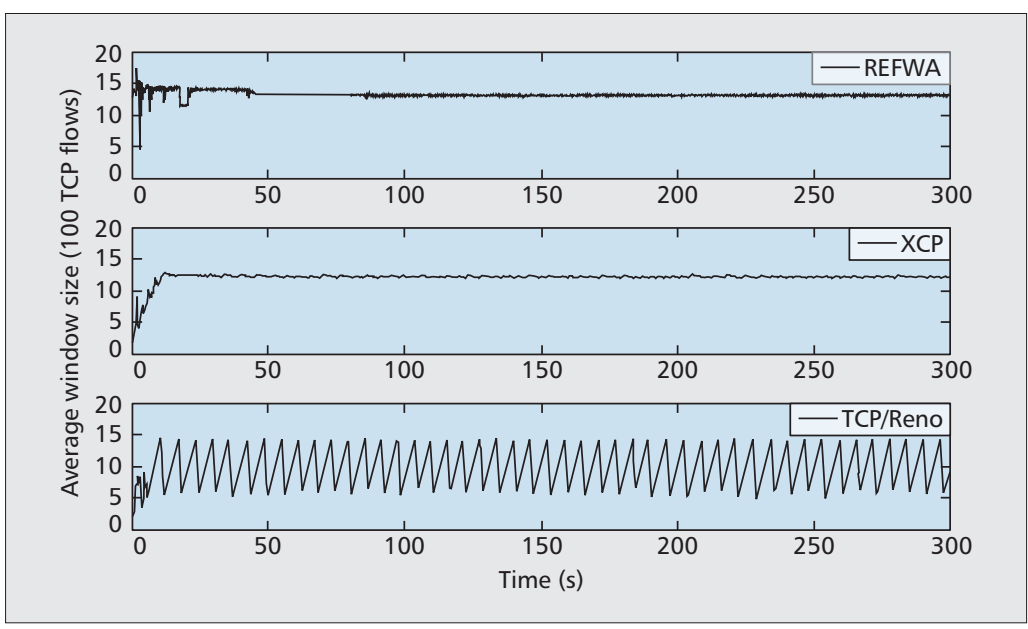

Figure 2. The average window size for REFWA, TCP, and XCP.

separate segments - end terminal to IGW, a segment traversing the satellite network, and the final segment between the IGW and the remote end terminal (Fig. 1). The use of the protocol pertains to the segment traversing the satellites. The other two segments will employ control mechanisms optimized for their characteristics (wireless or wired). Furthermore, the necessary intelligence required for coordination between the used data transmission mechanisms will be added to IGWs to ensure reliable delivery of data while meeting the end-to-end QoS requirements.

To illustrate the idea with more clarity, we have conducted simulations using NS-2. The window-based nature of REFWA is replaced by a rate-based one as explained earlier. The performance of the modified REFWA is compared against that of XCP and TCP as shown in Fig. 2. We tested the system under homogeneous traffic conditions using 100 heavy FTP sources during $300 \mathrm{~s}$, a duration long enough to capture and study the behavior of our proposed transport protocol. At the beginning of the simulation, the hosts behind each satellite terminal are activated randomly following a uniform distribution ranging from 10 to $100 \mathrm{~ms}$.

Figure 2 shows the average window size for the three protocols. From the figure, it is clear that the average window size, when REFWA is in use, converges immediately to the optimal window value. In contrast, in case of $\mathrm{XCP}$, it takes $15 \mathrm{~s}$ before the system reaches its optimal window. With TCP/Reno, the system oscillates around the optimal value without reaching a steady state. The simulation results also demonstrate the good performance of the modified REFWA as it achieves the highest goodput. Indeed, REFWA outperforms XCP and TCP/Reno by 6.93 and 20.90 percent, respectively, in terms of goodput.

For the sake of further transmission efficiency and better QoS, short RTT connections should be established via the terrestrial wireless network. Indeed, terminals communicating with nearby users do not have to drain their energy to connect directly to satellites. Long RTT connections can be set via satellites. Different tech- niques can be used for periodic monitoring of the network conditions. IGWs will be constantly updated with feedback on network dynamics. Based on this feedback and the RTT of connections, IGWs decide the path for communication - either via the satellites or via only the terrestrial network. For this purpose and in order to blur the separation between the satellite and terrestrial domains, there is need for exchange of state information (e.g., instant link loads) between the two domains. A hierarchical architecture of gateways can be considered. With this regard, the number of levels of this hierarchy, the size of each level, the amount of control traffic that should be exchanged and the length of the monitoring interval time should be decided in a way that enhances the accuracy in the assessment of network dynamics while minimizing the overhead in terms of signaling messages. Such a context-aware routing scheme will yield a better load balancing over the entire network and will enhance the end-to-end (E2E) QoS [4].

In the considered interworked satellite/terrestrial network, the IGW also provides the interface for any service/content provider who desires to provide service/content over both the terrestrial and satellite networks. If the provided data is bursty in nature (e.g., video data) and the targeted population of users is potential, it will be highly useful to send data from the provider to users using satellite channels. In such a communication scenario (Fig. 3), a service subscriber issues a request for a particular data/video title to the IGW via the terrestrial network (wireless or wired). The IGW informs the service provider of the request, and the latter allocates the necessary resources to satisfy the user's request. To ensure reliable transmission of data (depending on the underlying transport protocol) the client keeps acknowledging successful receptions of data to the IGW via acknowledgment (ACK) packets sent over the terrestrial network. IF the ACK packets are delayed or lost, the overall network performance may be impacted. Adding intelligence in terms of an adequate delayed acknowledgment mechanism along with a robust error recovery mechanism to IGWs can help cope with these issues.

\section{Resource Allocation and Management}

In light of the limited resources of any powerful network, QoS can be maintained only via efficient resource management/allocation mechanisms. In the case of converged satellite/ terrestrial networks, devising an efficient resource allocation method is a highly challenging task due to the fluctuating nature of the wireless links. Indeed, in DVB-S2 networks, for instance, a novel satellite-tailored adaptive coding and modulation (ACM) technique is introduced to cope with the wireless channel fluctuations. ACM renders the resource reservation process even more difficult since the channel capacity changes frequently as the channel experiences noisy periods.

While there are many approaches to solving resource management issues in networks, one that is suitable when there are limited and costly resources is connection admission control (CAC). 
A large library of CAC schemes has been proposed in the literature. These techniques can be classified as either resource-reservation-based or statistical-multiplexing-based schemes. Resource reservation CAC systems have some known scalability issues and may often lead to self-induced congestion due to the heavy resource reservation process. Besides, static reservation falls short of satisfying the flexibility requirements of typical network operators. Furthermore, statistical multiplexing CAC approaches cannot completely eliminate congestion during some peak noisy periods. However, they enable resource sharing between users and yield reduced waste of resources.

This feature renders statistical multiplexing schemes more suitable for converged satellite/ terrestrial networks. Several research works have devised different CAC schemes to guarantee a reasonable QoS level under different network conditions [5]. A common shortcoming of these schemes resides in their inefficiency in dealing with the varying nature of the physical layer capacity of the satellite network.

The authors' research work presented in [6] takes into account the satellite channel fluctuations and presents an interesting CAC mechanism that also ensures fairness among terminals competing for the capacity of the same satellite channel. A shortcoming of the proposed approach lies in its lack of a bandwidth allocation mechanism and multiservice support. Indeed, CAC should be exerted in conjunction with a bandwidth allocation mechanism, especially in converged satellite/terrestrial networks where the link capacity may vary as a result of the ACM mechanism. In this case, combined action among various layers, a cross-layer approach, of the networks is likely to improve the performance of the overall system by protecting, for example, prioritized flows from packet drops during congestion events. In this area of research, there is a particular interest in the development of a cross-layer bandwidth allocation mechanism that can assist CAC and further enhance its functionality.

For this purpose, we suggest using a channel prediction mechanism based on the least mean square (LMS) algorithm to tackle the excessive delay incurred by the feedback in satellite networks. Based on the proposed model, a self-configuring mechanism to cope with variable network conditions is derived. From this crosslayer approach, both optimized bandwidth allocation and guaranteed per-class QoS are expected.

To illustrate the idea, we have conducted simulations using Opnet. We test the system under homogeneous traffic conditions using FTP sources during $1500 \mathrm{~s}$. In the first phase of the simulation, the system is maintained free from noise. At the beginning of the second phase of the simulation (i.e., $t=245 \mathrm{~s}$ ), a source of noise is introduced, which directly impacts the signalto-noise ratio (SNR), which decreases by approximately $2 \mathrm{~dB}$. At the third phase of the simulation (i.e., $t=790 \mathrm{~s}$ ), another source of noise is introduced. Finally, at the last phase, which starts at $t=1000 \mathrm{~s}$, the sources of noise are eliminated successively at $t=1000 \mathrm{~s}$ and $t=$

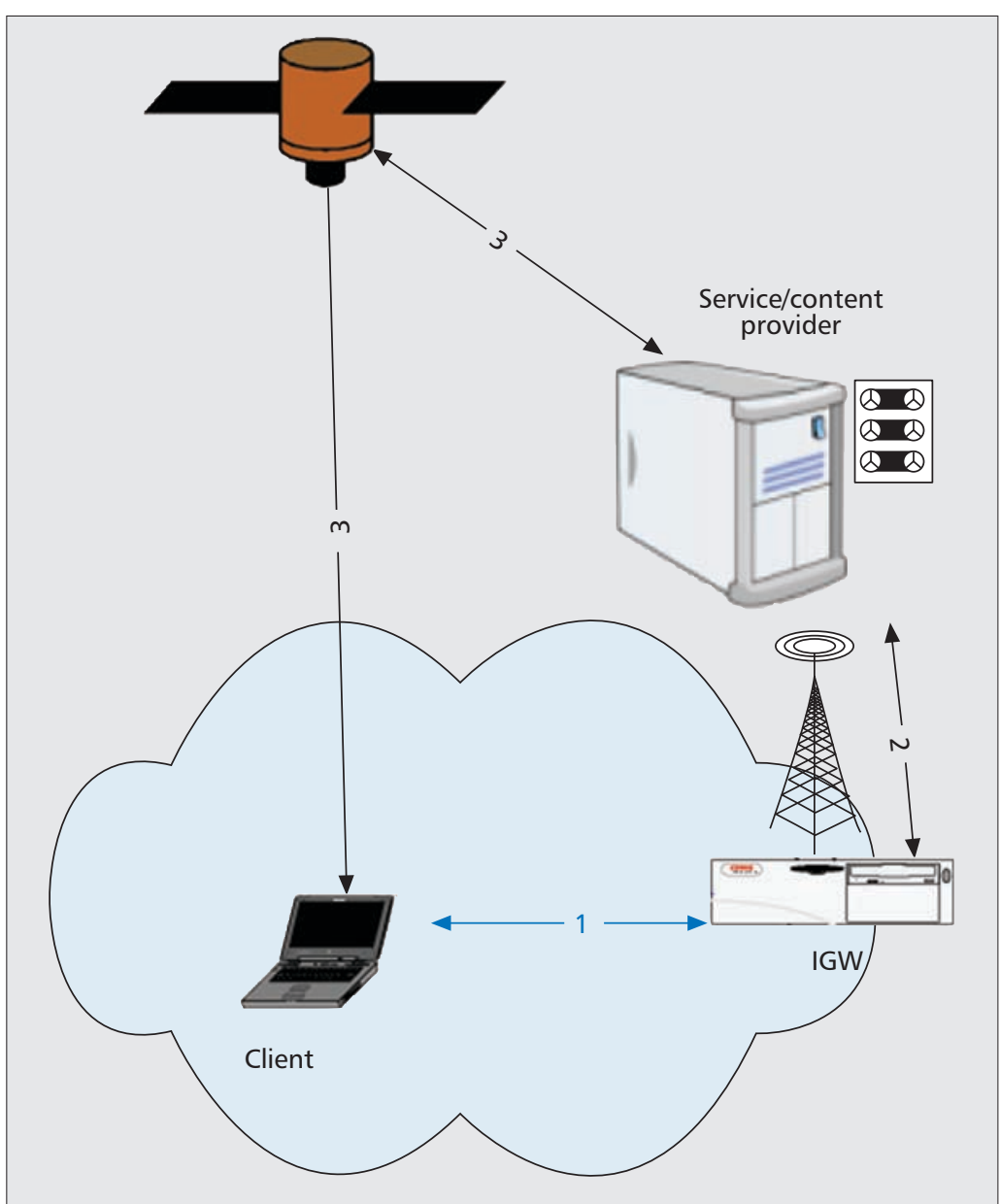

Figure 3. Service delivery pattern.

$1210 \mathrm{~s}$. This performance evaluation scenario allows us to see how quickly and accurately our scheme adapts to both degrading and improving conditions.

Figure 4 shows the instantaneous SNR experienced by a satellite terminal vs. the predicted SNR. In the first stage of the simulation, we observe that the estimation error is relatively important. This is principally due to the random initialization used in LMS. After 105 s, we clearly see that the estimation becomes more precise, which demonstrates the effectiveness of our proposed prediction mechanism. At this point, the accuracy of the proposed algorithm is approximately 98.5 percent.

Figure 5 indicates the performance of the proposed mechanism in terms of throughput. The figure clearly shows that the bandwidth manager using predicted SNR values, which allow selecting the appropriate modulation and code rate, outperforms the conventional approach. As a consequence, the experienced throughput is increased by approximately 4.6 percent.

The proposed cross-layer CAC mechanism, which relies on predicted SNR values, protects the network from congestion while maintaining a good trade-off between bandwidth utilization and end-to-end delay. These performances are particularly interesting for the provision of delay-sensitive and bandwidth-intensive applica- 


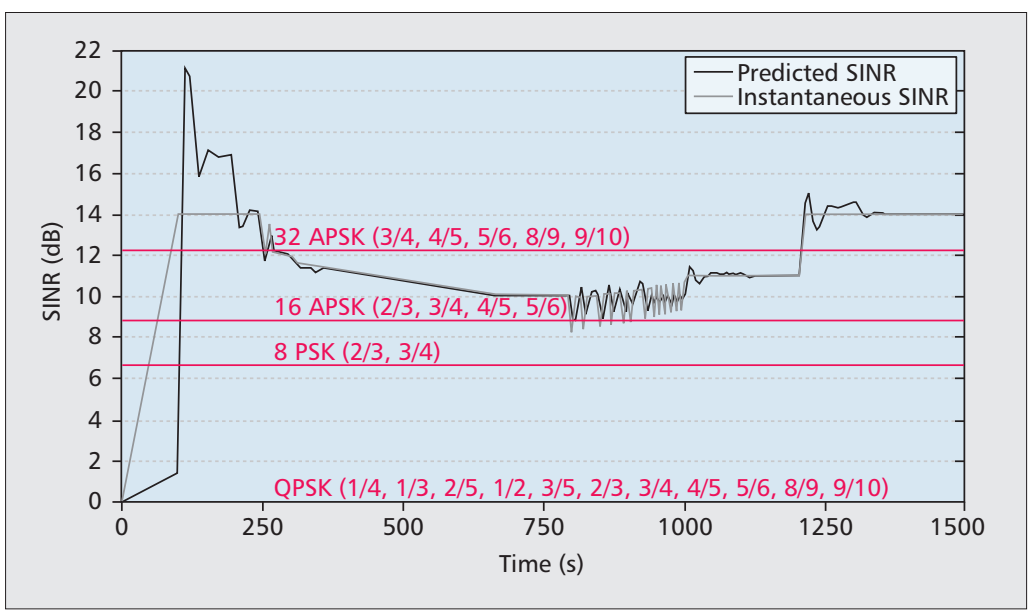

Figure 4. The instantaneous $S N R$ vs. the predicted $S N R$.

tions over converged satellite/terrestrial networks.

\section{MOBility Management}

The success of any communication system hinges on its ability to provide acceptable QoS. In the context of mobile environments, QoS provisioning depends in turn on an efficient management strategy for mobility. In satellite networks, mobility management is a challenging task. Indeed, supporting continuous communication over satellite systems may require changing spot beams (and links in LEO/MEO systems) as well as the IP address of the communication endpoints. Thus, both link-layer and network-layer handovers are required for satellite networking. In case of non-geostationary (NGEO) systems, mobility management becomes more complex as both the satellite network and mobile users are on the move.

In satellite networks, handovers can be broadly classified into two categories: network-link handover and link-layer handover. The former occurs when one of the communication endpoints changes its IP address due to motion of satellites or mobility of the user terminal. The latter occurs when one or more links between the end terminals change. It consists of satellite handover, ISL handover, and spot beam handover. Spot beam handover is the most common type of handover. They occur frequently due to the small area covered by spot beams and the mobility of users (or high speed of NGEO satellites). In this article, we initially focus on spot beam handovers and then extend the study to other handover types.

Spot Beam Handover Management - For better frequency utilization, the footprint of an individual satellite is divided into smaller cells, called spot beams. To ensure uninterrupted ongoing communications, a current communication link should be handed off to the next spot beam when needed. A spot beam handover involves the release of the communication link between the user and the current spot beam and acquiring a new link from the next spot beam to continue the communication. Due to the small area covered by spot beams, users' mobility, and high satellite speed in the case of NGEO systems, spot beam handovers are the most common type of handovers experienced in satellite systems.

Efficient management of handovers is particularly linked to the resource allocation problem discussed above. Indeed, the selection of a suitable policy for channel allocation can ensure channel availability during handover. Thus, channel allocation strategies and handover guarantee are the prime issues in managing handover requests. It should be noted that it is more desirable to ensure and guarantee smooth ongoing calls rather than block a newly arriving call. To solve the spot beam handover problem, several handover schemes have been proposed in the recent literature. A thorough survey on these techniques is available in [7].

Sophisticated network planning is required to assign more capacity to spot beams when a high traffic rate is expected. Statistical methods, coupled with a user behavior model and precise predictions of satellite tracks relative to the Earth surface, allow general characterization of the traffic load for a particular satellite or spot beam. Via a cross-layer design, this can help in anticipating imminent handover events and locating the new point of attachment to the satellite network [8]. While a cross-layer optimization can be implemented at either end devices or intermediate nodes in the network, such as IGWs, it is relatively easier and more feasible to implement changes at mobile nodes. Indeed, at the communication endpoint, the physical layer of a mobile host instantly measures the radio strength or link quality. When the mobile node moves into the overlapping area of two or more spot beams, and different signals are consequently detected by the physical and data link layers, a warning message notifying of an imminent handoff event along with a list of the new possible spot beams are sent to the application layer. In case of multiple spot beams, the application layer refers to a set of tools to sort out the spot beam to which the mobile node is most likely going to be connected. Indeed, the application layer may use history on the user's mobility pattern to predict the new spot beam. Referring to a spatial conceptual map, along with the user's personal information, its current position, and its velocity heading, the application layer can make an accurate prediction of the most probable future spot beam. Prior contextual knowledge of the coverage area of the satellite network and the type of application can further increase the accuracy of the prediction. Once the next spot beam is determined, the mobile host informs the IGW of the next spot beam. Based on the current conditions (e.g., maximum number of free channels) of each spot beam, the IGW decides whether the call should be accepted or denied. If the handover cannot be made without degrading QoS of already existing users or causing network congestion, the IGW denies the handover request and sends an immediate negative acknowledgment to inform the mobile host that the request has been turned down. Simultaneously, a list of available spot beams can be sent along with the negative acknowledgment to induce the mobile host to hand over with another spot beam. The mecha- 
nisms by which IGWs admit or turn down handover requests (from a user) should be consistent with the underlying resource allocation strategy. An actual design and implementation of this context-aware cross-layer architecture at the mobile terminals and the intelligence required by IGWs to manage handovers define an interesting topic of research in this particular field of research.

Satellite and ISL Handover Management in NGEO Systems - The solution suggested above may also be highly interesting when it is put in the context of mobile satellite communication systems (e.g., LEO, MEO). In these systems, satellite handovers are more important as users need to first choose among different satellites and will then be served by the spot beam covering the user. In addition to the above solution, there is a need to develop complementary solutions that select the most suitable satellite for communications that can reduce bandwidth waste and call blocking probability, and also fulfill the QoS requirements. In regard to QoS, the application type should be taken into account in the satellite handover management strategy.

Another important issue in the context of converged terrestrial/satellite networks is how to manage network layer handovers. While there are IP-based mobility solutions which have already reached the marketplace, they are unsuitable for converged satellite/terrestrial networks. This is mainly because they result in a very large amount of signaling traffic when employed in a satellite context, due to the constant and rapid motion of the satellites. Consequently, alternative network-layer handover mechanisms are required.

\section{SEAMLESS CONNECTIVITY}

While in the above subsections the focus was on defining issues pertaining to congestion control, resource allocation, and mobility management in the converged satellite/terrestrial network, and devising possible solutions, in the remainder of this article we discuss possible scenarios for seamless use of the interworked satellite/terrestrial network and provide guidelines for their realization.

IMS-Based Service Delivery Archifecture - A possible solution for service integration between satellite and terrestrial networks can be based on IMS, which represents a key element in the satellite architecture, supporting seamless and universal access to personalized services. Indeed, the adoption of IMS will favor the rapid emergence of new secure services and will enable seamless provisioning of multimedia services. IMS provides a service delivery platform (SDP) on top of convergence network technologies. This will help in generating new revenues, reducing the complexity of the IWG while significantly decreasing the cost of the satellite network management, which impacts directly the satellite services cost. Additionally, IMS allows more efficient handling of the multicast and broadcast traffic initiated from the satellite terminals. The rest of this section describes how to handle multicast services using IMS-based architecture.

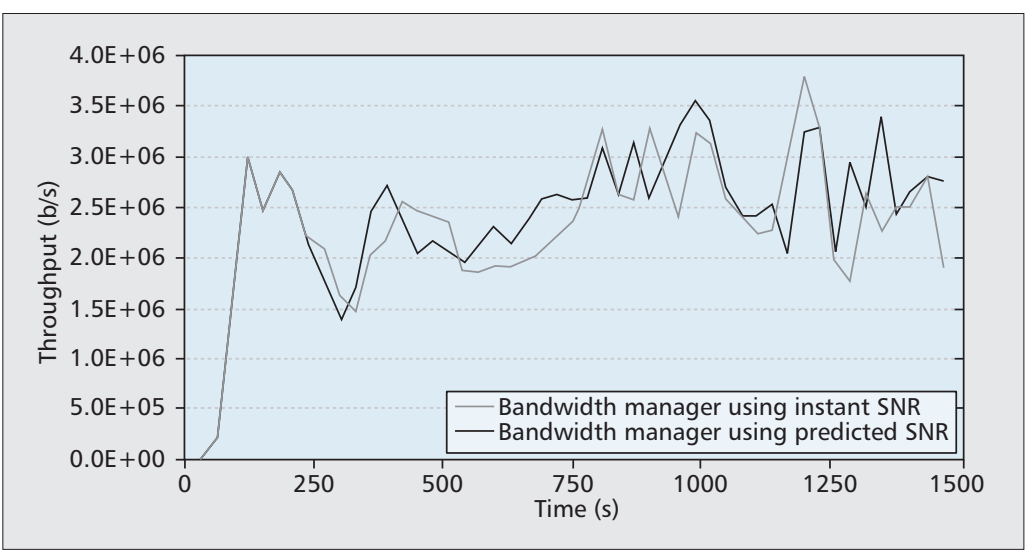

Figure 5. Throughput using instantaneous vs. predicted SNR.

Current mechanisms for delivering multicast services over satellite links use snooping (layer 2 ) or proxying (layer 3 ) to allow the delivery of IGMP/MLD membership messages to a satellite gateway over the air interface. A proxy and snooper do not change anything in the IGMP/ MLD messages but only forward the request further toward theIGW. In fact, over the satellite network, the broadcast property exists only on the forwarding link (i.e., the satellite return link provides only directional links). The host cannot listen to the signaling reports transmitted by other hosts on the return link. This leads them to individually send out their report, which generates excessive traffic. This would result in flooding and high latency problems.

Flooding occurs when many hosts (i.e., IGMP/MLD clients) reply to a broadcast request from the IGMP/MLD Querier sent out by the router to sense the presence of clients in each multicast group. As highlighted earlier, unlike LAN, the satellite return link does not provide a broadcast property but only a unidirectional connection. Typically, hosts cannot listen directly to replies from other hosts. Thus, all the hosts have to respond to the IGMP/MLD Querier after the expiration of their timer. Moreover, satellite multicast groups can be very large and very dynamic. This leads to a waste of bandwidth and CPU over the satellite link and the Gateway respectively.

Another important issue of IP multicast behavior over satellite networks is the latency in stopping transmission after the last host leaves a multicast group. The latency is the delay needed for the Querier to become aware that the multicast group is empty in order to stop multicast forwarding on it. The authors explain in [9] how to tackle the flooding and latency issues in providing multicast services. In this context, the IMS service delivery platform is used, which allows the IGMP messages to be aggregated and transmitted as a Session Initiation Protocol (SIP)based message for managing multicast groups. The argument for using SIP/IMS protocols is to allow hosts to join and leave a multicast group as IGMP does, verify the authentication and authorization of the user, signal any cryptographic context (e.g., using MIKEY), and easily support any future extension/augmentation that can be implemented in SIP. 
A possible solution

for service integration between satellite and terrestrial network can be

based on the IP

Multimedia

Subsystem that

represents a key

element in the

satellite architecture,

supporting seamless

and universal access

to personalised

services.

\section{CONCLUDING ReMARKS}

This article highlights some of the opportunities behind integrated satellite and terrestrial networks. For the realization of such converged networks, it addresses a number of issues pertaining to transmission efficiency, resource allocation and management, mobility management, and seamless connectivity. While the main objective of this article is to highlight the related issues and define new directions for the community of satellite researchers, it also suggests a number of solutions, as seen from the networking perspectives, that, once they are put together, form a complete context-aware end-to-end QoS approach that solves the aforementioned issues.

\section{REFERENCES}

[1] J. Farserotu and R. Prasad, "A Survey of Future Broadband Multimedia Satellite Systems, Issues and Trends," IEEE Commun. Mag., vol. 38, no. 6, 2000.

[2] T. Taleb, N. Kato, and Y. Nemoto, "REFWA: An Efficient and Fair Congestion Control Scheme for LEO Satellite Networks," IEEE/ACM Trans. Net. J., vol. 14, no. 5, Oct. 2006. pp. 1031-44

[3] M. Marchese, M. Rossi, and G. Morabito, "PETRA: Performance Enhancing Transport Architecture for Satellite Communications," IEEE JSAC, vol. 22, no. 2, Feb. 2004, pp. 320-22.

[4] T. Taleb et al., "Explicit Load Balancing Technique for NGEO Satellite IP Networks with On-Board Processing Capability," to appear, IEEE/ACM Trans. Net. J.

[5] R. Abi Fadel and S. Tomhe, "Connection Admission Control and Comparison of Two Differentiated Resources Allocations Schemes in a Low Earth Orbit Satellite Constellation" ACM Wireless Net. J., vol. 10, no. 10, May 2004.

[6] Y. H. Aoul and T. Taleb, "An Adaptive Fuzzy-Based CAC Scheme for Uplink and Downlink Congestion Control in Converged IP and DVB-S2 Networks," IEEE Trans. Wireless Commun., to appear.

[7] P. K. Chowdhury, M. Atiquzzaman, and W. Ivancic, "Handover Schemes in Satellite Networks: State-of-theArt and Future Research Directions," IEEE Commun. Surveys \& Tutorials, vol. 8, no. 4, 4th qtr. 2006.

[8] T. Taleb et al., "A Cross-Layer Approach for an Efficient Delivery of TCP/RTP-Based Multimedia Applications in Heterogeneous Wireless Networks," IEEE Trans. Vehic. Tech., vol. 57, no. 6, Nov. 2008, pp. 3801-14.

[9] T. Ahmed et al. "IMS-based IP Multicast Service Delivery over Satellite Network" 8th IEEE PIMRC '07, Sept. 2007.

\section{BIOGRAPHY}

TARIK TALEB [S`04, M’05, SM’10] (tarik.taleb@nw.neclab.eu) is currently working as a senior researcher at NEC Europe Ltd, Heidelberg, Germany. Prior to his current position until March 2009, he worked as am assistant professor at the Graduate School of Information Sciences, Tohoku University, Japan. From October 2005 to March 2006 he worked as a research fellow with the Intelligent Cosmos Research Institute, Sendai, Japan. He received his B.E. degree in information engineering with distinction, and M.Sc. and Ph.D. degrees in information sciences from GSIS, Tohoku University, in 2001, 2003, and 2005, respectively.

YASSINE HADJADJ (hadjadj@ieee.org) is an associate professor at the University of Rennes 1, France, where he is also a member of the IRISA Laboratory. He received a B.Sc. in computer engineering with high honors from SENIA University, Oran, Algeria, in 1999. He received his Master's and $\mathrm{Ph} . \mathrm{D}$. degrees in computer science from the University of Versailles, France, in 2002 and 2007, respectively. He was an assistant professor at the University of Versailles from 2005 to 2007, where he was involved in several national and European projects such as NMS, IST-ATHENA, and IST-IMOSAN. He was also a post-doctoral fellow at the University of Lille 1 and a research fellow, under the EUFP6 EIF Marie Curie Action, at the National University of Dublin where he was involved in the DOM'COM and IST-CARMEN projects, which aim at developing mixed Wi-Fi/WiMAX wireless mesh networks to support carrier grade services. His main research interests concern the fields of wireless networking, multimedia streaming, congestion control and QoS provisioning, and satellite communications. His work on multimedia and wireless communications has led to more than 25 technical papers in journals and international conference proceedings.

TOUfIK AHMED (tad@labri.fr) is a professor at Institut Polytechnique de Bordeaux (IPB) in the ENSEIRB-MATMECA School of Engineering. He is doing his research activities in CNRS LaBRI Lab, UMR 5800 at the University of Bordeaux 1. $\mathrm{He}$ received a B.Sc. in computer engineering with high honors from the National Institute of Computer Science, Algiers, Algeria, in 1999, and M.Sc. and Ph.D degrees in computer science from the University of Versailles, France, in 2000 and 2003, respectively. In November 2008 he obtained his Habilitation à Diriger des Recherches degree from the University of Bordeaux 1 on adaptive streaming and control of video QoS over wired/wireless IP networks and $\mathrm{P} 2 \mathrm{P}$ architectures. He was a visiting scientist at the School of Computer Science of the University of Waterloo in 2002 and a research fellow at PRiSM laboratory of the University of Versailles until 2004. His main research activities concern QoS for multimedia wired and wireless networks, end-to-end signaling protocols, P2P networks, and wireless sensor networks. His work on QoS and video delivery has led to many publications in major journals and conferences. 